\title{
On the solvability of general boundary value problems for systems of nonlinear impulsive equations with finite and fixed points of impulse actions
}

\author{
Malkhaz Ashordia ${ }^{1,2^{*}}$, Goderdzi Ekhvaia ${ }^{2}$ and Nestan Kekelia ${ }^{2}$
}

*Correspondence: ashord@rmi.ge

${ }^{1}$ A. Razmadze Mathematical Institute of I. Javakhishvili Tbilisi State University, 6 Tamarashvili St. Tbilisi, 0177, Georgia

${ }^{2}$ Sukhumi State University,

12 Politkovskaia St., Tbilisi, 0186,

Georgia

\begin{abstract}
General nonlocal boundary value problems are considered for systems of impulsive equations with finite and fixed points of impulses. Sufficient conditions are established for the solvability and unique solvability of these problems, among them effective spectral conditions.

MSC: $34 \mathrm{~B} 37$

Keywords: nonlocal boundary value problems; nonlinear systems; impulsive equations; solvability; unique solvability; effective conditions
\end{abstract}

\section{Statement of the problem and formulation of the results}

In the present paper, we consider the system of nonlinear impulsive equations with a finite number of impulse points

$$
\begin{aligned}
& \frac{d x}{d t}=f(t, x) \quad \text { almost everywhere on }[a, b] \backslash\left\{\tau_{1}, \ldots, \tau_{m_{0}}\right\}, \\
& x\left(\tau_{l}+\right)-x\left(\tau_{l}-\right)=I_{l}\left(x\left(\tau_{l}\right)\right) \quad\left(l=1, \ldots, m_{0}\right)
\end{aligned}
$$

with the general boundary value condition

$$
h(x)=0,
$$

where $a<\tau_{1}<\cdots<\tau_{m_{0}}<b$ (we will assume $\tau_{0}=a$ and $\tau_{m_{0}+1}=b$, if necessary), $-\infty<$ $a<b<+\infty, m_{0}$ is a natural number, $f$ belongs to Carathéodory class $\operatorname{Car}\left([a, b] \times \mathbb{R}^{n}, \mathbb{R}^{n}\right)$, $I_{l}: \mathbb{R}^{n} \rightarrow \mathbb{R}^{n}\left(l=1, \ldots, m_{0}\right)$ are continuous operators, and $h: C_{s}\left([a, b], \mathbb{R}^{n} ; \tau_{1}, \ldots, \tau_{m_{0}}\right) \rightarrow \mathbb{R}^{n}$ is a continuous, vector functional, nonlinear, in general.

In the paper sufficient conditions (among them effective sufficient) are given for solvability and unique solvability of the general nonlinear impulsive boundary value problem (1.1), (1.2); (1.3). We established the Conti-Opial type theorems for the solvability and unique solvability of this problem. Analogous problems are investigated in [1-5] (see also the references therein) for the general nonlinear boundary value problems for ordinary differen-

(c) 2014 Ashordia et al.; licensee Springer. This is an Open Access article distributed under the terms of the Creative Commons Attribution License (http://creativecommons.org/licenses/by/2.0), which permits unrestricted use, distribution, and reproduction in any medium, provided the original work is properly credited. 
tial and functional-differential systems, and in [6-10] (see also the references therein) for generalized ordinary differential systems.

Some results obtained in the paper are more general than known results even for the ordinary differential case.

Quite a number of issues of the theory of systems of differential equations with impulsive effect (both linear and nonlinear) have been studied sufficiently well (for a survey of the results on impulsive systems, see, e.g., [10-19] and references therein). But the abovementioned works, as is well known, do not contain the results obtained in the present paper.

Throughout the paper the following notation and definitions will be used.

$\mathbb{R}=]-\infty,+\infty\left[, \mathbb{R}_{+}=[0,+\infty[;[a, b](a, b \in \mathbb{R})\right.$ is a closed segment;

$\mathbb{R}^{n \times m}$ is the space of all real $n \times m$ matrices $X=\left(x_{i j}\right)_{i, j=1}^{n, m}$ with the norm

$$
\begin{aligned}
& \|X\|=\max _{j=1, \ldots, m} \sum_{i=1}^{n}\left|x_{i j}\right| ; \\
& |X|=\left(\left|x_{i j}\right|\right)_{i, j=1}^{n, m}, \quad[X]_{+}=\frac{|X|+X}{2} ; \\
& \mathbb{R}_{+}^{n \times m}=\left\{\left(x_{i j}\right)_{i, j=1}^{n, m}: x_{i j} \geq 0(i=1, \ldots, n ; j=1, \ldots, m)\right\} ; \\
& \mathbb{R}^{(n \times n) \times m}=\mathbb{R}^{n \times n} \times \cdots \times \mathbb{R}^{n \times n}(m \text { times }) ;
\end{aligned}
$$

$\mathbb{R}^{n}=\mathbb{R}^{n \times 1}$ is the space of all real column $n$-vectors $x=\left(x_{i}\right)_{i=1}^{n} ; \mathbb{R}_{+}^{n}=\mathbb{R}_{+}^{n \times 1}$;

if $X \in \mathbb{R}^{n \times n}$, then $X^{-1}$, $\operatorname{det} X$ and $r(X)$ are, respectively, the matrix inverse to $X$, the determinant of $X$, and the spectral radius of $X ; I_{n \times n}$ is the identity $n \times n$ matrix;

$\bigvee_{a}^{b}(X)$ is the variation of the matrix function $X:[a, b] \rightarrow \mathbb{R}^{n \times m}$, i.e., the sum of variations of the latter's components; $V(X)(t)=\left(v\left(x_{i j}\right)(t)\right)_{i, j=1}^{n, m}$, where $v\left(x_{i j}\right)(a)=0, v\left(x_{i j}\right)(t)=\bigvee_{a}^{t}\left(x_{i j}\right)$ for $a<t \leq b$;

$X(t-)$ and $X(t+)$ are the left and the right limits of the matrix function $X:[a, b] \rightarrow \mathbb{R}^{n \times m}$ at the point $t$ (we will assume $X(t)=X(a)$ for $t \leq a$ and $X(t)=X(b)$ for $t \geq b$, if necessary);

$$
\|X\|_{s}=\sup \{\|X(t)\|: t \in[a, b]\}
$$

$\mathrm{BV}\left([a, b], \mathbb{R}^{n \times m}\right)$ is the set of all matrix functions of bounded variation $X:[a, b] \rightarrow \mathbb{R}^{n \times m}$ (i.e., such that $\left.\bigvee_{a}^{b}(X)<+\infty\right)$;

$C([a, b], D)$, where $D \subset \mathbb{R}^{n \times m}$, is the set of all continuous matrix functions $X:[a, b] \rightarrow D$;

$C\left([a, b], D ; \tau_{1}, \ldots, \tau_{m_{0}}\right)$ is the set of all matrix functions $X:[a, b] \rightarrow D$, having the one sided limits $X\left(\tau_{l^{-}}\right)\left(l=1, \ldots, m_{0}\right)$ and $X\left(\tau_{l^{+}}\right)\left(l=1, \ldots, m_{0}\right)$, whose restrictions to an arbitrary closed interval $[c, d]$ from $[a, b] \backslash\left\{\tau_{1}, \ldots, \tau_{m_{0}}\right\}$ belong to $C([c, d], D)$;

$C_{s}\left([a, b], \mathbb{R}^{n \times m} ; \tau_{1}, \ldots, \tau_{m_{0}}\right)$ is the Banach space of all matrix functions $X \in C([a, b]$, $\left.\mathbb{R}^{n \times m} ; \tau_{1}, \ldots, \tau_{m_{0}}\right)$ with the norm $\|X\|_{s}$;

$\widetilde{C}([a, b], D)$, where $D \subset \mathbb{R}^{n \times m}$, is the set of all absolutely continuous matrix functions $X:[a, b] \rightarrow D$;

$\widetilde{C}\left([a, b], D ; \tau_{1}, \ldots, \tau_{m_{0}}\right)$ is the set of all matrix functions $X:[a, b] \rightarrow D$, having the one sided limits $X\left(\tau_{l^{-}}\right)\left(l=1, \ldots, m_{0}\right)$ and $X\left(\tau_{l}+\right)\left(l=1, \ldots, m_{0}\right)$, whose restrictions to an arbitrary closed interval $[c, d]$ from $[a, b] \backslash\left\{\tau_{k}\right\}_{k=1}^{m_{0}}$ belong to $\widetilde{C}([c, d], D)$. 
If $B_{1}$ and $B_{2}$ are normed spaces, then an operator $g: B_{1} \rightarrow B_{2}$ (nonlinear, in general) is positive homogeneous if $g(\lambda x)=\lambda g(x)$ for every $\lambda \in \mathbb{R}_{+}$and $x \in B_{1}$.

The inequalities between the matrices are understood componentwise.

An operator $\varphi: C\left([a, b], \mathbb{R}^{n \times m} ; \tau_{1}, \ldots, \tau_{m_{0}}\right) \rightarrow \mathbb{R}^{n}$ is called nondecreasing if for every $x, y \in C\left([a, b], \mathbb{R}^{n \times m} ; \tau_{1}, \ldots, \tau_{m_{0}}\right)$ such that $x(t) \leq y(t)$ for $t \in[a, b]$ the inequality $\varphi(x) \leq \varphi(y)$ holds.

A matrix function is said to be continuous, nondecreasing, integrable, etc., if each of its components is.

$L([a, b], D)$, where $D \subset \mathbb{R}^{n \times m}$, is the set of all measurable and integrable matrix functions $X:[a, b] \rightarrow D$.

If $D_{1} \subset \mathbb{R}^{n}$ and $D_{2} \subset \mathbb{R}^{n \times m}$, then $\operatorname{Car}\left([a, b] \times D_{1}, D_{2}\right)$ is the Carathéodory class, i.e., the set of all mappings $F=\left(f_{k j}\right)_{k, j=1}^{n, m}:[a, b] \times D_{1} \rightarrow D_{2}$ such that:

(a) the function $f_{k j}(\cdot, x):[a, b] \rightarrow D_{2}$ is measurable for every $x \in D_{1}$;

(b) the function $f_{k j}(t, \cdot): D_{1} \rightarrow D_{2}$ is continuous for almost all $t \in[a, b]$, and

$$
\begin{aligned}
& \sup \left\{\left|f_{k j}(\cdot, x)\right|: x \in D_{0}\right\} \in L([a, b], \mathbb{R}) \\
& \quad \text { for every compact } D_{0} \subset D_{1}(k=1, \ldots, n ; j=1, \ldots, m) .
\end{aligned}
$$

$\operatorname{Car}^{0}\left([a, b] \times D_{1}, D_{2}\right)$ is the set of all mappings $F=\left(f_{k j}\right)_{k, j=1}^{n, m}:[a, b] \times D_{1} \rightarrow D_{2}$ such that the functions $f_{k j}(\cdot, x(\cdot))(k=1, \ldots, n ; j=1, \ldots, m)$ are measurable for every vector function $x:[a, b] \rightarrow \mathbb{R}^{n}$ with bounded variation.

By a solution of the impulsive system (1.1), (1.2) we understand a vector function $x \in$ $\widetilde{C}\left([a, b], \mathbb{R}^{n} ; \tau_{1}, \ldots, \tau_{m_{0}}\right)$, continuous from the left, satisfying both the system (1.1) a.e. on $[a, b] \backslash\left\{\tau_{1}, \ldots, \tau_{m_{0}}\right\}$ and the relation (1.2) for every $k \in\left\{1, \ldots, m_{0}\right\}$.

Definition 1.1 Let $\ell: C_{s}\left([a, b], \mathbb{R}^{n} ; \tau_{1}, \ldots, \tau_{m_{0}}\right) \rightarrow \mathbb{R}^{n}$ be a linear continuous operator, and let $\ell_{0}: C_{s}\left([a, b], \mathbb{R}^{n} ; \tau_{1}, \ldots, \tau_{m_{0}}\right) \rightarrow \mathbb{R}_{+}^{n}$ be a positive homogeneous operator. We say that a pair $\left(P,\left\{J_{l}\right\}_{l=1}^{m_{0}}\right)$, consisting of a matrix function $P \in \operatorname{Car}\left([a, b] \times \mathbb{R}^{n}, \mathbb{R}^{n \times n}\right)$ and a finite sequence of continuous operators $J_{l}=\left(J_{l i}\right)_{i=1}^{n}: \mathbb{R}^{n} \rightarrow \mathbb{R}^{n}\left(l=1, \ldots, m_{0}\right)$, satisfy the Opial condition with respect to the pair $\left(\ell, \ell_{0}\right)$ if:

(a) there exist a matrix function $\Phi \in L\left([a, b], \mathbb{R}_{+}^{n \times n}\right)$ and constant matrices $\Psi_{l} \in \mathbb{R}^{n \times n}$ $\left(l=1, \ldots, m_{0}\right)$ such that

$$
|P(t, x)| \leq \Phi(t) \quad \text { a.e. on }[a, b], x \in \mathbb{R}^{n}
$$

and

$$
\left|J_{l}(x)\right| \leq \Psi_{l} \quad \text { for } x \in \mathbb{R}^{n}\left(l=1, \ldots, m_{0}\right) ;
$$

(b)

$$
\operatorname{det}\left(I_{n \times n}+G_{l}\right) \neq 0 \quad\left(l=1, \ldots, m_{0}\right)
$$

and the problem

$$
\frac{d x}{d t}=A(t) x \quad \text { a.e. on }[a, b] \backslash\left\{\tau_{1}, \ldots, \tau_{m_{0}}\right\},
$$




$$
\begin{aligned}
& x\left(\tau_{l}+\right)-x\left(\tau_{l}-\right)=G_{l} x\left(\tau_{l}\right) \quad\left(l=1, \ldots, m_{0}\right) \\
& |\ell(x)| \leq \ell_{0}(x)
\end{aligned}
$$

has only the trivial solution for every matrix function $A \in L\left([a, b], \mathbb{R}^{n \times n}\right)$ and constant matrices $G_{l}\left(l=1, \ldots, m_{0}\right)$ for which there exists a sequence $y_{k} \in \widetilde{C}\left([a, b], \mathbb{R}^{n} ; \tau_{1}, \ldots, \tau_{m_{0}}\right)$ $(k=1,2, \ldots)$ such that

$$
\lim _{k \rightarrow+\infty} \int_{a}^{t} P\left(\tau, y_{k}(\tau)\right) d \tau=\int_{a}^{t} A(\tau) d \tau \quad \text { uniformly on }[a, b]
$$

and

$$
\lim _{k \rightarrow+\infty} J_{l}\left(y_{k}\left(\tau_{l}\right)\right)=G_{l} \quad\left(l=1, \ldots, m_{0}\right) .
$$

Remark 1.1 Note that, due to the condition (1.5), the condition (1.6) holds if

$$
\left\|\Psi_{l}\right\|<1 \quad\left(l=1, \ldots, m_{0}\right) .
$$

Below, we will assume that $f=\left(f_{i}\right)_{i=1}^{n} \in \operatorname{Car}\left([a, b] \times \mathbb{R}^{n}, \mathbb{R}^{n}\right)$ and, in addition, $f\left(\tau_{l}, x\right)$ can be arbitrary for $x \in \mathbb{R}^{n}$ and $l=1, \ldots, m_{0}$.

Theorem 1.1 Let the conditions

$$
\begin{aligned}
& \|f(t, x)-P(t, x) x\| \leq \alpha(t,\|x\|) \quad \text { a.e. on }[a, b] \backslash\left\{\tau_{1}, \ldots, \tau_{m_{0}}\right\}, x \in \mathbb{R}^{n}, \\
& \left\|I_{l}(x)-J_{l}(x) x\right\| \leq \beta_{l}(\|x\|) \quad \text { for } x \in \mathbb{R}^{n}\left(l=1, \ldots, m_{0}\right)
\end{aligned}
$$

and

$$
|h(x)-\ell(x)| \leq \ell_{0}(x)+\ell_{1}\left(\|x\|_{s}\right) \quad \text { for } x \in C_{s}\left([a, b], \mathbb{R}^{n} ; \tau_{1}, \ldots, \tau_{m_{0}}\right)
$$

hold, where $\ell: C_{s}\left([a, b], \mathbb{R}^{n} ; \tau_{1}, \ldots, \tau_{m_{0}}\right) \rightarrow \mathbb{R}^{n}$ and $\ell_{0}: C_{s}\left([a, b], \mathbb{R}^{n} ; \tau_{1}, \ldots, \tau_{m_{0}}\right) \rightarrow \mathbb{R}_{+}^{n}$ are, respectively, linear continuous and positive homogeneous continuous operators, the pair $\left(P,\left\{J_{l}\right\}_{l=1}^{m_{0}}\right)$ satisfies the Opial condition with respect to the pair $\left(\ell, \ell_{0}\right) ; \alpha \in \operatorname{Car}([a, b] \times$ $\left.\mathbb{R}_{+}, \mathbb{R}_{+}\right)$is a function nondecreasing in the second variable, and $\beta_{l} \in C\left(\mathbb{R}_{+}, \mathbb{R}_{+}\right)(l=$ $\left.1, \ldots, m_{0}\right)$ and $\ell_{1} \in C\left(\mathbb{R}_{+}, \mathbb{R}_{+}^{n}\right)$ are nondecreasing, respectively, functions and vector functions such that

$$
\lim _{\rho \rightarrow+\infty} \frac{1}{\rho}\left(\left\|\ell_{1}(\rho)\right\|+\int_{a}^{b} \alpha(t, \rho) d t+\sum_{l=1}^{m_{0}} \beta_{l}(\rho)\right)=0 .
$$

Then the problem (1.1), (1.2); (1.3) is solvable.

Theorem 1.2 Let the conditions (1.12)-(1.14),

$$
P_{1}(t) \leq P(t, x) \leq P_{2}(t) \quad \text { a.e. on }[a, b] \backslash\left\{\tau_{1}, \ldots, \tau_{m_{0}}\right\}, x \in \mathbb{R}^{n}
$$


and

$$
J_{1 l} \leq J_{l}(x) \leq J_{2 l} \quad \text { for } x \in \mathbb{R}^{n}\left(l=1, \ldots, m_{0}\right)
$$

hold, where $P \in \operatorname{Car}^{0}\left([a, b] \times \mathbb{R}^{n}, \mathbb{R}^{n \times n}\right), P_{i} \in L\left([a, b], \mathbb{R}^{n \times n}\right)(i=1,2), J_{i l} \in \mathbb{R}^{n \times n}(i=$ 1,$\left.2 ; l=1, \ldots, m_{0}\right), \ell: C_{s}\left([a, b], \mathbb{R}^{n} ; \tau_{1}, \ldots, \tau_{m_{0}}\right) \rightarrow \mathbb{R}^{n}$ and $\ell_{0}: C_{s}\left([a, b], \mathbb{R}^{n} ; \tau_{1}, \ldots, \tau_{m_{0}}\right) \rightarrow$ $\mathbb{R}_{+}^{n}$ are, respectively, linear continuous and positive homogeneous continuous operators; $\alpha \in \operatorname{Car}\left([a, b] \times \mathbb{R}_{+}, \mathbb{R}_{+}\right)$is a function nondecreasing in the second variable, and $\beta_{l} \in$ $C\left([a, b], \mathbb{R}_{+}\right)\left(l=1, \ldots, m_{0}\right)$ and $\ell_{1} \in C\left(\mathbb{R}_{+}, \mathbb{R}_{+}^{n}\right)$ are nondecreasing, respectively, functions and vector function such that the condition (1.15) holds. Let, moreover, the condition (1.6) hold and the problem (1.7), (1.8); (1.9) have only the trivial solution for every matrix function $A \in L\left([a, b], \mathbb{R}^{n \times n}\right)$ and constant matrices $G_{l} \in \mathbb{R}^{n \times n}\left(l=1, \ldots, m_{0}\right)$ such that

$$
P_{1}(t) \leq A(t) \leq P_{2}(t) \quad \text { a.e. on }[a, b] \backslash\left\{\tau_{1}, \ldots, \tau_{m_{0}}\right\}, x \in \mathbb{R}^{n}
$$

and

$$
J_{1 l} \leq G_{l} \leq J_{2 l} \quad \text { for } x \in \mathbb{R}^{n}\left(l=1, \ldots, m_{0}\right) .
$$

Then the problem (1.1), (1.2); (1.3) is solvable.

Remark 1.2 Theorem 1.2 is interesting only in the case when $P \notin \operatorname{Car}\left([a, b] \times \mathbb{R}^{n}, \mathbb{R}^{n \times n}\right)$, because the theorem immediately follows from Theorem 1.1 in the case when $P \in$ $\operatorname{Car}\left([a, b] \times \mathbb{R}^{n}, \mathbb{R}^{n \times n}\right)$.

Theorem 1.3 Let the conditions (1.14),

$$
\left|f(t, x)-P_{0}(t) x\right| \leq Q(t)|x|+q(t,\|x\|) \quad \text { a.e. on }[a, b] \backslash\left\{\tau_{1}, \ldots, \tau_{m_{0}}\right\}, x \in \mathbb{R}^{n}
$$

and

$$
\left|I_{l}(x)-J_{0 l} x\right| \leq H_{l}|x|+h_{l}(\|x\|) \quad \text { for } x \in \mathbb{R}^{n}\left(l=1, \ldots, m_{0}\right)
$$

hold, where $P_{0} \in L\left([a, b], \mathbb{R}^{n \times n}\right), Q \in L\left([a, b], \mathbb{R}_{+}^{n \times n}\right), J_{0 l}$ and $H_{l} \in \mathbb{R}^{n \times n}\left(l=1, \ldots, m_{0}\right)$ are constant matrices, $\ell: C_{s}\left([a, b], \mathbb{R}^{n} ; \tau_{1}, \ldots, \tau_{m_{0}}\right) \rightarrow \mathbb{R}^{n}$ and $\ell_{0}: C_{s}\left([a, b], \mathbb{R}^{n} ; \tau_{1}, \ldots, \tau_{m_{0}}\right) \rightarrow$ $\mathbb{R}_{+}^{n}$ are, respectively, linear continuous and positive homogeneous continuous operators; $q \in \operatorname{Car}\left([a, b] \times \mathbb{R}_{+}, \mathbb{R}_{+}^{n}\right)$ is a vector function nondecreasing in the second variable, and $h_{l} \in$ $C\left(\mathbb{R}_{+}, \mathbb{R}_{+}^{n}\right)\left(l=1, \ldots, m_{0}\right)$ and $\ell_{1} \in C\left(\mathbb{R}_{+}, \mathbb{R}_{+}^{n}\right)$ are nondecreasing vector functions such that

$$
\lim _{\rho \rightarrow+\infty} \frac{1}{\rho}\left(\left\|\ell_{1}(\rho)\right\|+\int_{a}^{b}\|q(t, \rho)\| d t+\sum_{l=1}^{m_{0}}\left\|h_{l}(\rho)\right\|\right)=0
$$

Let, moreover, the conditions

$$
\operatorname{det}\left(I_{n \times n}+J_{0 l}\right) \neq 0 \quad\left(l=1, \ldots, m_{0}\right)
$$


and

$$
\left\|H_{l}\right\| \cdot\left\|\left(I_{n \times n}+J_{0 l}\right)^{-1}\right\|<1 \quad\left(l=1, \ldots, m_{0}\right)
$$

hold and the system of impulsive inequalities

$$
\begin{aligned}
& \left|\frac{d x}{d t}-P_{0}(t) x\right| \leq Q(t)|x| \quad \text { a.e. on }[a, b] \backslash\left\{\tau_{1}, \ldots, \tau_{m_{0}}\right\}, \\
& \left|x\left(\tau_{l}+\right)-x\left(\tau_{l}-\right)-J_{0 l} x\left(\tau_{l}\right)\right| \leq H_{l}\left|x\left(\tau_{l}\right)\right| \quad\left(l=1, \ldots, m_{0}\right)
\end{aligned}
$$

have only the trivial solution under the condition (1.9). Then the problem (1.1), (1.2); (1.3) is solvable.

Corollary 1.1 Let the conditions

$$
\begin{aligned}
& \|f(t, x)-P(t) x\| \leq \alpha(t,\|x\|) \quad \text { a.e. on }[a, b] \backslash\left\{\tau_{1}, \ldots, \tau_{m_{0}}\right\}, x \in \mathbb{R}^{n}, \\
& \left\|I_{l}(x)-J_{l} x\right\| \leq \beta_{l}(\|x\|) \quad \text { for } x \in \mathbb{R}^{n}\left(l=1, \ldots, m_{0}\right)
\end{aligned}
$$

and

$$
\|h(x)-\ell(x)\| \leq \gamma\left(\|x\|_{s}\right) \quad \text { for } x \in C_{s}\left([a, b], \mathbb{R}^{n} ; \tau_{1}, \ldots, \tau_{m_{0}}\right)
$$

hold, where $P \in L\left([a, b], \mathbb{R}^{n \times n}\right), J_{l} \in \mathbb{R}^{n \times n}\left(l=1, \ldots, m_{0}\right)$ are constant matrices, $\ell: C_{s}([a, b]$, $\left.\mathbb{R}^{n} ; \tau_{1}, \ldots, \tau_{m_{0}}\right) \rightarrow \mathbb{R}^{n}$ is a linear continuous operator, $\alpha \in \operatorname{Car}\left([a, b] \times \mathbb{R}_{+}, \mathbb{R}_{+}\right)$is a function nondecreasing in the second variable, and $\beta_{l} \in C\left(\mathbb{R}_{+}, \mathbb{R}_{+}\right)\left(l=1, \ldots, m_{0}\right)$ and $\gamma \in C\left(\mathbb{R}_{+}, \mathbb{R}_{+}\right)$ are nondecreasing functions such that

$$
\lim _{\rho \rightarrow+\infty} \frac{1}{\rho}\left(\gamma(\rho)+\int_{a}^{b} \alpha(t, \rho) d t+\sum_{l=1}^{m_{0}} \beta_{l}(\rho)\right)=0 .
$$

Let, moreover,

$$
\operatorname{det}\left(I_{n \times n}+J_{l}\right) \neq 0 \quad\left(l=1, \ldots, m_{0}\right)
$$

and the impulsive system

$$
\begin{aligned}
& \frac{d x}{d t}=P(t) x \quad \text { a.e. on }[a, b] \backslash\left\{\tau_{1}, \ldots, \tau_{m_{0}}\right\}, \\
& x\left(\tau_{l}+\right)-x\left(\tau_{l}-\right)=J_{l} x\left(\tau_{l}\right) \quad\left(l=1, \ldots, m_{0}\right)
\end{aligned}
$$

have only the trivial solution under the condition

$$
\ell(x)=0 .
$$

Then the problem (1.1), (1.2); (1.3) is solvable. 
For every matrix function $X \in L\left([a, b], \mathbb{R}^{n \times n}\right)$ and a sequence of constant matrices $Y_{k} \in$ $\mathbb{R}^{n \times n}\left(k=1, \ldots, m_{0}\right)$ we introduce the operators

$$
\begin{aligned}
& {\left[\left(X, Y_{1}, \ldots, Y_{m_{0}}\right)(t)\right]_{0}=I_{n} \quad \text { for } a \leq t \leq b,} \\
& {\left[\left(X, Y_{1}, \ldots, Y_{m_{0}}\right)(a)\right]_{i}=O_{n \times n} \quad(i=1,2, \ldots)} \\
& {\left[\left(X, Y_{1}, \ldots, Y_{m_{0}}\right)(t)\right]_{i+1}} \\
& \quad \int_{a}^{t} X(\tau)\left[\left(X, Y_{1}, \ldots, Y_{m_{0}}\right)(\tau)\right]_{i} d \tau+\sum_{a \leq \tau_{l}<t} Y_{l}\left[\left(X, Y_{1}, \ldots, Y_{m_{0}}\right)\left(\tau_{l}\right)\right]_{i} \\
& \quad \text { for } a<t \leq b(i=0,1, \ldots) .
\end{aligned}
$$

Corollary 1.2 Let the conditions (1.26)-(1.30) hold, where

$$
\ell(x) \equiv \int_{a}^{b} d \mathcal{L}(t) \cdot x(t)
$$

$P \in L\left([a, b], \mathbb{R}^{n \times n}\right), J_{l} \in \mathbb{R}^{n \times n}\left(l=1, \ldots, m_{0}\right)$ are constant matrices, $\mathcal{L} \in \mathrm{BV}\left([a, b], \mathbb{R}^{n \times n}\right), \alpha \in$ $\operatorname{Car}\left([a, b] \times \mathbb{R}_{+}, \mathbb{R}_{+}\right)$is a function nondecreasing in the second variable, and $\beta_{l} \in C\left(\mathbb{R}_{+}, \mathbb{R}_{+}\right)$ $\left(l=1, \ldots, m_{0}\right)$ and $\gamma \in C\left(\mathbb{R}_{+}, \mathbb{R}_{+}\right)$are nondecreasing functions. Let, moreover, there exist natural numbers $k$ and $m$ such that the matrix

$$
M_{k}=-\sum_{i=0}^{k-1} \int_{a}^{b} d \mathcal{L}(t) \cdot\left[\left(P, J_{l}, \ldots, J_{m_{0}}\right)(t)\right]_{i}
$$

is nonsingular and

$$
r\left(M_{k, m}\right)<1
$$

where the operators $\left[\left(P, J_{1}, \ldots, J_{m_{0}}\right)(t)\right]_{i}(i=0,1, \ldots)$ are defined by $(1.33)$, and

$$
\begin{aligned}
M_{k, m}= & {\left[\left(|P|,\left|J_{1}\right|, \ldots,\left|J_{m_{0}}\right|\right)(b)\right]_{m}+\sum_{i=0}^{m-1}\left[\left(|P|,\left|J_{1}\right|, \ldots,\left|J_{m_{0}}\right|\right)(b)\right]_{i} } \\
& \times \int_{a}^{b} d V\left(M_{k}^{-1} \mathcal{L}\right)(t) \cdot\left[\left(|P|,\left|J_{1}\right|, \ldots,\left|J_{m_{0}}\right|\right)(t)\right]_{k}
\end{aligned}
$$

Then the problem (1.1), (1.2); (1.3) is solvable.

Corollary 1.3 Let the conditions (1.26)-(1.30) hold, where

$$
\ell(x) \equiv \sum_{j=1}^{n_{0}} \mathcal{L}_{j} x\left(t_{j}\right)
$$

$P \in L\left([a, b], \mathbb{R}^{n \times n}\right), J_{l} \in \mathbb{R}^{n \times n}\left(l=1, \ldots, m_{0}\right)$ are constant matrices, $t_{j} \in[a, b]$ and $\mathcal{L}_{j} \in \mathbb{R}^{n \times n}$ $\left(j=1, \ldots, n_{0}\right), \alpha \in \operatorname{Car}\left([a, b] \times \mathbb{R}_{+}, \mathbb{R}_{+}\right)$is a function nondecreasing in the second variable, and $\beta_{l} \in C\left(\mathbb{R}_{+}, \mathbb{R}_{+}\right)\left(l=1, \ldots, m_{0}\right)$ and $\gamma \in C\left(\mathbb{R}_{+}, \mathbb{R}_{+}\right)$are nondecreasing functions. Let, 
moreover, the constant matrices $J_{l}\left(l=1, \ldots, m_{0}\right)$ be pairwise permutable, and let the matrix function P satisfy the Lappo-Danilevskil condition, i.e.

$$
P(t) \int_{a}^{t} P(\tau) d \tau=\int_{a}^{t} P(\tau) d \tau \cdot P(t) \quad \text { for } t \in[a, b]
$$

and

$$
P(t) J_{l}=J_{l} P(t) \quad \text { a.e. on }[a, b]\left(l=1, \ldots, m_{0}\right) .
$$

Then the condition

$$
\operatorname{det}\left(\sum_{j=1}^{n_{0}} \mathcal{L}_{j} \exp \left(P\left(t_{j}\right)\right) \cdot \prod_{a \leq \tau_{l}<t_{j}}\left(I_{n \times n}+J_{l}\right)\right) \neq 0
$$

guarantees the solvability of the problem (1.1), (1.2); (1.3).

Corollary 1.4 Let the conditions (1.26)-(1.30) and (1.35) hold, where $P \in L\left([a, b], \mathbb{R}^{n \times n}\right)$, $J_{l} \in \mathbb{R}^{n \times n}\left(l=1, \ldots, m_{0}\right)$ are constant matrices, $t_{j} \in[a, b]$ and $\mathcal{L}_{j} \in \mathbb{R}^{n \times n}\left(j=1, \ldots, n_{0}\right), \alpha \in$ $\operatorname{Car}\left([a, b] \times \mathbb{R}_{+}, \mathbb{R}_{+}\right)$is a function nondecreasing in the second variable, and $\beta_{l} \in C\left(\mathbb{R}_{+}, \mathbb{R}_{+}\right)$ $\left(l=1, \ldots, m_{0}\right)$ and $\gamma \in C\left(\mathbb{R}_{+}, \mathbb{R}_{+}\right)$are nondecreasing functions. Let, moreover, there exist natural numbers $k$ and $m$ such that the matrix

$$
M_{k}=\sum_{j=1}^{n_{0}} \sum_{i=0}^{k-1} \mathcal{L}_{j}\left[\left(P_{0}, J_{l}, \ldots, J_{m_{0}}\right)\left(t_{j}\right)\right]_{i}
$$

is nonsingular and the inequality (1.34) holds, where

$$
\begin{aligned}
M_{k, m}= & {\left[\left(|P|,\left|J_{l}\right|, \ldots,\left|J_{m_{0}}\right|\right)(b)\right]_{m}+\left(\sum_{i=0}^{m-1}\left[\left(|P|,\left|J_{l}\right|, \ldots,\left|J_{m_{0}}\right|\right)(b)\right]_{i}\right) } \\
& \times \sum_{j=1}^{n_{0}}\left|M_{k}^{-1} \mathcal{L}_{j}\right| \cdot\left[\left(|P|,\left|J_{l}\right|, \ldots,\left|J_{m_{0}}\right|\right)\left(t_{j}\right)\right]_{k} .
\end{aligned}
$$

Then the problem (1.1), (1.2); (1.3) is solvable.

Corollary 1.5 Let the conditions (1.26)-(1.30) and (1.35) hold, where $P \in L\left([a, b], \mathbb{R}^{n \times n}\right)$, $J_{l} \in \mathbb{R}^{n \times n}\left(l=1, \ldots, m_{0}\right)$ are constant matrices, $t_{j} \in[a, b]$ and $\mathcal{L}_{j} \in \mathbb{R}^{n \times n}\left(j=1, \ldots, n_{0}\right), \alpha \in$ $\operatorname{Car}\left([a, b] \times \mathbb{R}_{+}, \mathbb{R}_{+}\right)$is a function nondecreasing in the second variable, and $\beta_{l} \in C\left(\mathbb{R}_{+}, \mathbb{R}_{+}\right)$ $\left(l=1, \ldots, m_{0}\right)$ and $\gamma \in C\left(\mathbb{R}_{+}, \mathbb{R}_{+}\right)$are nondecreasing functions. Let, moreover,

$$
\operatorname{det}\left(\sum_{j=1}^{n_{0}} \mathcal{L}_{j}\right) \neq 0
$$

hold and

$$
r\left(\mathcal{L}_{0} \cdot V(A)(b)\right)<1,
$$


where

$$
\mathcal{L}_{0}=I_{n \times n}+\left|\left(\sum_{j=1}^{n_{0}} \mathcal{L}_{j}\right)^{-1}\right| \cdot \sum_{j=1}^{n_{0}}\left|\mathcal{L}_{j}\right| \text { and } A=\int_{a}^{b}|P(t)| d t+\sum_{l=1}^{m_{0}}\left|J_{l}\right| .
$$

Then the problem (1.1), (1.2); (1.3) is solvable.

Theorem 1.4 Let the conditions (1.22), (1.23),

$$
\begin{aligned}
& \left|f(t, x)-f(t, y)-P_{0}(t)(x-y)\right| \leq Q(t)|x-y| \\
& \quad \text { a.e. on }[a, b] \backslash\left\{\tau_{1}, \ldots, \tau_{m_{0}}\right\}, x, y \in \mathbb{R}^{n}, \\
& \left|I_{l}(x)-I_{l}(y)-J_{0 l}(x-y)\right| \leq H_{l}|x-y| \quad \text { for } x, y \in \mathbb{R}^{n}\left(l=1, \ldots, m_{0}\right)
\end{aligned}
$$

and

$$
|h(x)-h(y)-\ell(x-y)| \leq \ell_{0}(x-y) \quad \text { for } x, y \in \mathrm{BV}\left([a, b], \mathbb{R}^{n}\right)
$$

hold, where $P \in L\left([a, b], \mathbb{R}^{n \times n}\right), Q \in L\left([a, b], \mathbb{R}_{+}^{n \times n}\right), J_{0 l}$ and $H_{l} \in \mathbb{R}^{n \times n}\left(l=1, \ldots, m_{0}\right)$ are constant matrices, $\ell: C_{s}\left([a, b], \mathbb{R}^{n} ; \tau_{1}, \ldots, \tau_{m_{0}}\right) \rightarrow \mathbb{R}^{n}$ and $\ell_{0}: C_{s}\left([a, b], \mathbb{R}^{n} ; \tau_{1}, \ldots, \tau_{m_{0}}\right) \rightarrow$ $\mathbb{R}_{+}^{n}$ are, respectively, linear continuous and positive homogeneous continuous operators. Let, moreover, the system of impulsive inequalities (1.24), (1.25) have only the trivial solution under the condition (1.9). Then the problem (1.1), (1.2); (1.3) is uniquely solvable.

\section{Auxiliary propositions}

Lemma 2.1 Let $Y, Y_{k} \in \mathrm{BV}\left([a, b], \mathbb{R}^{n \times m}\right)(k=1,2, \ldots)$ be such that

$$
\lim _{k \rightarrow+\infty} Y_{k}(t)=Y(t) \quad \text { for } t \in[a, b]
$$

and

$$
\left\|Y_{k}(t)-Y_{k}(s)\right\| \leq l_{k}+\|g(t)-g(s)\| \quad \text { for } a \leq s \leq t \leq b(k=1,2, \ldots),
$$

where $l_{k} \geq 0, l_{k} \rightarrow 0$ as $k \rightarrow+\infty$, and $g:[a, b] \rightarrow \mathbb{R}^{n}$ is a nondecreasing vector function. Then

$$
\lim _{k \rightarrow+\infty}\left\|Y_{k}-Y\right\|_{s}=0
$$

The proof of Lemma 2.1 is given in [9].

Lemma 2.2 (Lemma on a priori estimates) Let the subsets $\mathcal{S} \subset L\left([a, b], \mathbb{R}^{n \times n}\right)$ and $\mathcal{D} \subset$ $\mathbb{R}^{(n \times n) \times m_{0}}$, and a positive homogeneous continuous operator $g: C_{s}\left([a, b], \mathbb{R}^{n} ; \tau_{1}, \ldots, \tau_{m_{0}}\right) \rightarrow$ $\mathbb{R}^{n}$ be such that:

(a) there exist a matrix function $\Phi \in L\left([a, b], \mathbb{R}_{+}^{n \times n}\right)$ and a constant matrix $\Psi \in \mathbb{R}_{+}^{n \times n}$ such that

$$
|A(t)| \leq \Phi(t) \quad \text { a.e. on }[a, b], x \in \mathbb{R}^{n}
$$


for every $A \in \mathcal{S}$, and

$$
\left|G_{l}\right| \leq \Psi \quad\left(l=1, \ldots, m_{0}\right) \text { for } \mathfrak{G}=\left(G_{l}\right)_{l=1}^{m_{0}} \in \mathcal{D}
$$

(b) the condition (1.6) holds and the system (1.7), (1.8) has only the trivial solution under the condition

$$
g(x) \leq 0
$$

for every matrix function $A \in \mathcal{S}$ and constant matrices $G_{1}, \ldots, G_{m_{0}}$ such that $\mathfrak{G}=$ $\left(G_{l}\right)_{l=1}^{m_{0}} \in \mathcal{D}$;

(c) if $A_{k} \in \mathcal{S}(k=1,2, \ldots), \mathfrak{G}_{k}=\left(G_{k l}\right)_{l=1}^{m_{0}} \in \mathcal{D}(k=1,2, \ldots), A \in L\left([a, b], \mathbb{R}^{n \times n}\right)$ and $\mathfrak{G}=$ $\left(G_{l}\right)_{l=1}^{m_{0}}$ are such that

$$
\lim _{k \rightarrow+\infty} \int_{a}^{t} A_{k}(\tau) d \tau=\int_{a}^{t} A(\tau) d \tau \quad \text { uniformly on }[a, b]
$$

and

$$
\lim _{k \rightarrow+\infty} G_{k l}=G_{l} \quad\left(l=1, \ldots, m_{0}\right)
$$

then $A \in \mathcal{S}$ and $\mathfrak{G}=\left(G_{l}\right)_{l=1}^{m_{0}} \in \mathcal{D}$. Then there exists a positive number $\rho_{0}$ such that

$$
\begin{aligned}
\|x\|_{s} \leq & \rho_{0}\left[\left\|[g(x)]_{+}\right\|\right. \\
& \left.+\sup \left\{\left\|x(t)-x(a)-\int_{a}^{t} A(\tau) x(\tau) d \tau-\sum_{\tau_{l} \in[a, t[} G_{l} x\left(\tau_{l}\right)\right\|: t \in[a, b]\right\}\right] \\
& \text { for } x \in \widetilde{C}\left([a, b], \mathbb{R}^{n} ; \tau_{1}, \ldots, \tau_{m_{0}}\right), A \in \mathcal{S}, \mathfrak{G}=\left(G_{l}\right)_{l=1}^{m_{0}} \in \mathcal{D} .
\end{aligned}
$$

Proof Let us assume that the statement of the lemma is not true. Then for every natural $k$ there exist a matrix function $A_{k} \in \mathcal{S}$, a constant matrix $\mathfrak{G}_{k}=\left(G_{k l}\right)_{l=1}^{m_{0}} \in \mathcal{D}$, and a vector function $x_{k} \in \widetilde{C}\left([a, b], \mathbb{R}^{n} ; \tau_{1}, \ldots, \tau_{m_{0}}\right)$ such that

$$
\begin{aligned}
\left\|x_{k}\right\|_{s}> & k\left[\left\|\left[g\left(x_{k}\right)\right]_{+}\right\|+\sup \left\{\| x_{k}(t)-x_{k}(a)-\int_{a}^{t} A_{k}(\tau) x_{k}(\tau) d \tau\right.\right. \\
& \left.\left.-\sum_{\tau_{l} \in[a, t[} G_{k l} x_{k}\left(\tau_{l}\right) \|: t \in[a, b]\right\}\right] .
\end{aligned}
$$

Let

$$
\begin{aligned}
& \tilde{x}_{k}(t)=\frac{1}{\left\|x_{k}\right\|_{s}} x_{k}(t) \quad \text { for } t \in[a, b](k=1,2, \ldots), \\
& q_{k}(t)=\widetilde{x}_{k}^{\prime}(t)-A_{k}(t) \widetilde{x}_{k}(t) \quad \text { for a.a. } t \in[a, b] \backslash\left\{\tau_{1}, \ldots, \tau_{m_{0}}\right\}(k=1,2, \ldots),
\end{aligned}
$$

and

$$
h_{k l}=\widetilde{x}_{k}\left(\tau_{l}+\right)-\widetilde{x}_{k}\left(\tau_{l}-\right)-G_{k l} \tilde{x}_{k}\left(\tau_{l}\right) \quad\left(l=1, \ldots, m_{0}, k=1,2, \ldots\right) .
$$


Let, moreover,

$$
B_{k}(t)=\int_{a}^{t} A_{k}(\tau) d \tau \quad \text { for } t \in[a, b](k=1,2, \ldots) .
$$

Then

$$
\begin{aligned}
& \left\|\tilde{x}_{k}\right\|_{s}=1 \quad(k=1,2, \ldots), \\
& \left\|\left[g\left(\tilde{x}_{k}\right)\right]_{+}\right\|<\frac{1}{k} \quad(k=1,2, \ldots)
\end{aligned}
$$

and

$$
\begin{aligned}
& \left\|\int_{a}^{t} q_{k}(\tau) d \tau+\sum_{\tau_{l} \in[a, t[} h_{k l}\right\|<\frac{1}{k} \\
& \quad \text { for } t \in[a, b](k=1,2, \ldots) \text {, and }\left\|h_{k l}\right\|<\frac{2}{k}\left(l=1, \ldots, m_{0} ; k=1,2, \ldots\right) .
\end{aligned}
$$

On the other hand, by the estimate (a) we have

$$
\left|B_{k}(t)-B_{k}(s)\right| \leq \int_{s}^{t} \Phi(\tau) d \tau \quad \text { for } a \leq s<t \leq b(k=1,2, \ldots)
$$

Therefore, by the Arzelá-Ascoli lemma we can assume without loss of generality that the sequence $B_{k}(k=1,2, \ldots)$ converges uniformly on $[a, b]$, and the sequence $G_{k l}(k=1,2, \ldots)$ converges for every $l \in\left\{1, \ldots, m_{0}\right\}$.

Let

$$
B(t)=\lim _{k \rightarrow+\infty} B_{k}(t) \quad \text { and } \lim _{k \rightarrow+\infty} G_{k l}=G_{l} \text { for } t \in[a, b]\left(l=1, \ldots, m_{0}\right) .
$$

It is evident that the matrix function $B$ is absolutely continuous. Therefore,

$$
B(t)=\int_{a}^{t} A(\tau) d \tau \quad \text { for } t \in[a, b]
$$

where $A \in L\left([a, b], \mathbb{R}^{n \times n}\right)$. From this and (2.6), by the condition (c) we have $A \in \mathcal{S}$ and $\mathfrak{G}=\left(G_{l}\right)_{l=1}^{m_{0}} \in \mathcal{D}$.

According to $(2.3)$ we can assume that the sequence $\tilde{x}_{k}(a)(k=1,2, \ldots)$ converges. It is evident that the function $\tilde{x}_{k}$ is a solution of the system

$$
\begin{aligned}
& \frac{d x}{d t}=A_{k}(t) x+q_{k}(t) \quad \text { a.e. on }[a, b] \backslash\left\{\tau_{1}, \ldots, \tau_{m_{0}}\right\}, \\
& x\left(\tau_{l}+\right)-x\left(\tau_{l}-\right)=G_{k l} x\left(\tau_{l}\right)+h_{k l} \quad\left(l=1, \ldots, m_{0}\right)
\end{aligned}
$$

for every natural $k$. Using now Theorem 1.2 of the paper [2], from the conditions (a), (2.5), and (2.6) it follows that

$$
\lim _{k \rightarrow+\infty}\left\|\tilde{x}_{k}-x\right\|_{s}=0
$$


where $x$ is a solution of the system (1.7), (1.8) under the condition

$$
x(a)=c_{0},
$$

and

$$
c_{0}=\lim _{k \rightarrow+\infty} \tilde{x}_{k}(a)
$$

Take into account (2.4) and (2.7), we conclude $g(x) \leq 0$. So that $x$ is a solution of the problem (1.7), (1.8); (2.1). Consequently, by the condition (b) we have $x(t) \equiv 0$. But this contradicts the condition (2.3). The lemma is proved.

\section{Proof of the main results}

Proof of Theorem 1.1 Let $g(x) \equiv|\ell(x)|-\ell_{0}(x), \mathcal{S} \subset L\left([a, b], \mathbb{R}^{n \times n}\right)$ and $\mathcal{D} \subset \mathbb{R}^{(n \times n) \times m_{0}}$ be, respectively, the sets of all matrix functions $A \in L\left([a, b], \mathbb{R}^{n \times n}\right)$ and constant matrix-vectors $\mathfrak{G}=\left(G_{k}\right)_{k=1}^{m_{0}}$, satisfying the condition (1.6), such that the conditions (1.10) and (1.11) hold for some sequence $y_{l} \in \widetilde{C}\left([a, b], \mathbb{R}^{n} ; \tau_{1}, \ldots, \tau_{m_{0}}\right)(l=1,2, \ldots)$. By virtue of Definition 1.1 the conditions (a), (b), (c) of Lemma 2.2 are fulfilled for the sets $\mathcal{S}$ and $\mathcal{D}$.

Let $\rho_{0}$ be the positive number appearing in the conclusion of Lemma 2.2. According to the condition (1.15) there exists a positive number $\rho_{1}$ such that

$$
\rho_{0}\left(\left\|\ell_{1}(\rho)\right\|+\int_{a}^{b} \alpha(t, \rho) d t+\sum_{l=1}^{m_{0}} \beta_{l}(\rho)\right)<\rho \quad \text { for } \rho \geq \rho_{1} \text {. }
$$

Assume

$$
\begin{aligned}
& q(t, x)=f(t, x)-P(t, x) x \text { and } H_{l}(x)=I_{l}(x)-J_{l}(x) x \\
& \text { for } t \in[a, b], x \in \mathbb{R}^{n}\left(l=1, \ldots, m_{0}\right) ; \\
& \chi(t)= \begin{cases}1 & \text { for } 0 \leq t<\rho_{1}, \\
2-\frac{t}{\rho_{1}} & \text { for } \rho_{1} \leq t<2 \rho_{1}, \\
0 & \text { for } t \geq 2 \rho_{1} ;\end{cases} \\
& \tilde{\ell}(x)=\chi\left(\|x\|_{s}\right)[\ell(x)-h(x)] ; \\
& \rho_{2}=2 \rho_{1}+\rho_{0} \sup \left\{\left\|\ell_{0}(y)\right\|+\left\|\ell_{1}\left(\|y\|_{s}\right)\right\|:\|y\|_{s} \leq 2 \rho_{1}\right\} ; \\
& \mathcal{U}=\left\{y \in C\left([a, b], \mathbb{R}^{n} ; \tau_{1}, \ldots, \tau_{m_{0}}\right):\|y\|_{s} \leq \rho_{2}\right\}
\end{aligned}
$$

and consider the auxiliary boundary value problem

$$
\begin{aligned}
& \frac{d x}{d t}=P(t, y(t)) x+q(t, y(t)) \quad \text { a.e. on }[a, b] \backslash\left\{\tau_{1}, \ldots, \tau_{m_{0}}\right\}, \\
& x\left(\tau_{l}+\right)-x\left(\tau_{l}-\right)=J_{l}\left(y\left(\tau_{l}\right)\right) x\left(\tau_{l}\right)+H_{l}\left(y\left(\tau_{l}\right)\right) \quad\left(l=1, \ldots, m_{0}\right) ; \\
& \ell(x)=\tilde{\ell}(y)
\end{aligned}
$$

for every $y \in \mathcal{U}$. 
According to the Opial condition the problem

$$
\begin{aligned}
& \frac{d x}{d t}=P(t, y(t)) x \quad \text { a.e. on }[a, b] \backslash\left\{\tau_{1}, \ldots, \tau_{m_{0}}\right\}, \\
& x\left(\tau_{l}+\right)-x\left(\tau_{l}-\right)=J_{l}\left(y\left(\tau_{l}\right)\right) x\left(\tau_{l}\right) \quad\left(l=1, \ldots, m_{0}\right) ; \\
& \ell(x)=0
\end{aligned}
$$

has only the trivial solution for every $y \in \mathcal{U}$.

Therefore, in view of Theorem 3.1 from [10] the problem (3.5), (3.6); (3.7) has a unique solution $x(t) \equiv \omega(y)(t)$. In addition, by (3.5), (3.6), and (3.7), it follows from Lemma 2.2 that

$$
\|\omega(y)\|_{s} \leq \rho_{0}\left(\|\tilde{\ell}(y)\|+\int_{a}^{b}\|q(t, y(t))\| d t+\sum_{l=1}^{m_{0}}\left\|H_{l}\left(y\left(\tau_{l}\right)\right)\right\|\right) .
$$

From this, due to (1.12), (1.13), and (3.2)-(3.4) we have

$$
\|\omega(y)\|_{s} \leq \rho_{0}\left(\|\widetilde{\ell}(y)\|+\int_{a}^{b} \alpha\left(t,\|y\|_{s}\right) d t+\sum_{l=1}^{m_{0}} \beta_{l}\left(\|y\|_{s}\right)\right) .
$$

On the other hand, taking into account the inequalities (1.14) and (3.1), the condition (3.8) implies

$$
\begin{aligned}
\|\omega(y)\|_{s} \leq & \rho_{0} \sup \left\{\left\|\ell_{0}(z)\right\|+\left\|\ell_{1}\left(\|z\|_{s}\right)\right\|:\|z\|_{s} \leq 2 \rho_{1}\right\} \\
& +\rho_{0}\left(\int_{a}^{b} \alpha\left(t,\|y\|_{s}\right) d t+\sum_{l=1}^{m_{0}} \beta_{l}\left(\|y\|_{s}\right)\right)<\rho_{2} \quad \text { for }\|y\|_{s} \leq 2 \rho_{1}
\end{aligned}
$$

and

$$
\|\omega(y)\|_{s} \leq \rho_{0}\left(\int_{a}^{b} \alpha\left(t,\|y\|_{s}\right) d t+\sum_{l=1}^{m_{0}} \beta_{l}\left(\|y\|_{s}\right)\right)<\|y\|_{s} \leq \rho_{2} \quad \text { for } 2 \rho_{1}<\|y\|_{s} \leq \rho_{2} .
$$

Thus $\omega(\mathcal{U}) \subset \mathcal{U}$. Further, due to Theorem 1 from [13] we conclude that the operator $\omega$ : $\mathcal{U} \rightarrow \mathcal{U}$ is continuous.

By (1.4), (1.5), (1.12), (1.13), and (3.2) we have

$$
\|\omega(y)(t)-\omega(y)(s)\| \leq \int_{s}^{t} \varphi_{0}(\tau) d \tau+\sum_{s \leq \tau_{l}<t} \psi_{l} \quad \text { for } a \leq s<t \leq b
$$

if $y \in \mathcal{U}$, where $\varphi_{0}(t)=\alpha\left(t, \rho_{2}\right)+\rho_{2}\|\Phi(t)\|$ and $\psi_{l}=\beta_{l}\left(\rho_{2}\right)+\rho_{2}\left\|\Psi_{l}\right\|$. So that, using the Arzelá-Ascoli lemma on the every closed interval $\left[\tau_{l-1}, \tau_{l}\right]\left(l=1, \ldots, m_{0}\right)$ we conclude that the set $\mathcal{U}$ is precompact.

According to the Schauder principle there exists $x \in \mathcal{U}$ such that

$$
x(t)=\omega(x)(t) \quad \text { for } a \leq t \leq b .
$$


From this, by virtue of (1.14) and (3.2)-(3.4), it follows that the function $x$ is a solution of the system (1.1), (1.2) satisfying the conditions

$$
\ell(x)=\tilde{\ell}(x)
$$

and

$$
|\ell(x)| \leq \ell_{0}(x)+\ell_{1}\left(\|x\|_{s}\right) .
$$

Due to Lemma 2.2 and inequalities (1.12), (1.13), (3.1), and (3.10) we have

$$
\|x\|_{s} \leq \rho_{0}\left(\left\|\ell_{1}\left(\|x\|_{s}\right)\right\|+\int_{a}^{b} \alpha\left(t,\|x\|_{s}\right) d t+\sum_{l=1}^{m_{0}} \beta_{l}\left(\|x\|_{s}\right)\right) \quad \text { and } \quad\|x\|_{s}<\rho_{1} .
$$

In fact, the first estimate immediately follows from Lemma 2.2 with regard to the conditions (1.12), (1.13), and (3.10). Now, if we assume that $\|x\|_{s} \geq \rho_{1}$ then by (3.1), for $\rho=\|x\|_{s}$, it will be

$$
\rho_{0}\left(\left\|\ell_{1}\left(\|x\|_{s}\right)\right\|+\int_{a}^{b} \alpha\left(t,\|x\|_{s}\right) d t+\sum_{l=1}^{m_{0}} \beta_{l}\left(\|x\|_{s}\right)\right)<\|x\|_{s}
$$

The obtained inequality contradicts the first estimate of (3.11).

In view of the estimate (3.11) from (3.3) and (3.4) we have $\widetilde{\ell}(x)=\ell(x)-h(x)$. Consequently, by (3.9) we conclude that the vector function $x$ satisfies the condition (1.3). The theorem is proved.

Proof of Theorem 1.2 Let $\mathcal{S}$ be the set of all matrix functions $A \in L\left([a, b] ; \mathbb{R}^{n \times n}\right)$ satisfying the inequalities (1.18), and let $\mathcal{D}$ be the set all constant matrices $\mathfrak{G}=\left(G_{l}\right)_{l=1}^{m_{0}}$ satisfying the condition (1.6) and the inequalities (1.19). It is evident that the conditions of Lemma 2.2 hold for these sets and the operator $g(x) \equiv|\ell(x)|-\ell_{0}(x)$.

Let $\rho_{0}$ be the number such that the conclusion of Lemma 2.2 is true. In view of (1.15) there exists a positive number $\rho_{1}$ such that the estimate (3.1) holds. Consider the impulsive system

$$
\begin{aligned}
& \frac{d x}{d t}=P_{1}(t) x+\chi(\|x\|)\left[f(t, x)-P_{1}(t) x\right] \quad \text { a.e. on } t \in[a, b] \backslash\left\{\tau_{1}, \ldots, \tau_{m_{0}}\right\}, \\
& x\left(\tau_{l}+\right)-x\left(\tau_{l}-\right)=J_{1 l} x\left(\tau_{l}\right)+\chi\left(\left\|x\left(\tau_{l}\right)\right\|\right)\left[I_{l}\left(x\left(\tau_{l}\right)\right)-J_{1 l} x\left(\tau_{l}\right)\right] \quad\left(l=1, \ldots, m_{0}\right),
\end{aligned}
$$

where $\chi$ is the function defined by (3.3). According to Theorem 1.1 the problem (3.12), (3.13); (1.3) is solvable since the pair $\left(P_{1},\left\{J_{1 l}\right\}_{l=1}^{m_{0}}\right)$ satisfies the Opial condition with respect to the pair $\left(\ell, \ell_{0}\right)$. Let $x$ be an arbitrary solution of this problem. Then

$$
\begin{aligned}
& x^{\prime}(t)-A(t) x(t)=\chi(\|x(t)\|)[f(t, x(t))-P(t, x(t)) x(t)] \quad \text { a.e. on } t \in[a, b] \backslash\left\{\tau_{1}, \ldots, \tau_{m_{0}}\right\}, \\
& x\left(\tau_{l^{+}}\right)-x\left(\tau_{l^{-}}\right)-G_{l} x\left(\tau_{l}\right)=\chi\left(\left\|x\left(\tau_{l}\right)\right\|\right)\left[I_{l}\left(x\left(\tau_{l}\right)\right)-J_{l}\left(x\left(\tau_{l}\right)\right) x\left(\tau_{l}\right)\right] \quad\left(l=1, \ldots, m_{0}\right),
\end{aligned}
$$

where

$$
A(t) \equiv P_{1}(t)+\chi(\|x(t)\|)\left[P(t, x(t))-P_{1}(t)\right],
$$


and

$$
G_{l}=J_{1 l}+\chi\left(\left\|x\left(\tau_{l}\right)\right\|\right)\left[J_{l}\left(x\left(\tau_{l}\right)\right)-J_{1 l}\right] \quad\left(l=1, \ldots, m_{0}\right) .
$$

On the other hand, by (1.16), (1.17), and (3.3) the matrix function $A$ and constant matrices $G_{l}\left(l=1, \ldots, m_{0}\right)$ satisfy, respectively, the inequalities (1.18) and (1.19). Therefore we have $A \in \mathcal{S}$ and $\mathfrak{G}=\left(G_{l}\right)_{l=1}^{m_{0}}$. Therefore, due to Lemma 2.2 and the inequalities (1.12)-(1.14) and (3.1), the estimate (3.11) is valid. But by (3.3) every solution of the system (3.12), (3.13) satisfying such an estimate is a solution of the system (1.1), (1.2), too. The theorem is proved.

Proof of Theorem 1.3 Let

$$
\begin{aligned}
& x=\left(x_{i}\right)_{i=1}^{n}, \quad f(t, x)=\left(f_{i}(t, x)\right)_{i=1}^{n}, \quad q(t, \rho)=\left(q_{i}(t, \rho)\right)_{i=1}^{n}, \\
& P_{0}(t)=\left(p_{0 i j}(t)\right)_{i, j=1}^{n}, \quad Q(t)=\left(q_{i j}(t)\right)_{i, j=1}^{n} ; \\
& I_{l}(x)=\left(\iota_{l i}(x)\right)_{i=1}^{n}, \quad J_{0 l}=\left(\gamma_{0 l i j}\right)_{i, j=1}^{n}, \quad H_{l}=\left(h_{l i j}\right)_{i, j=1}^{n}, \\
& h_{l}(\rho)=\left(h_{l i}(\rho)\right)_{i=1}^{n} \quad\left(l=1, \ldots, m_{0}\right) .
\end{aligned}
$$

Assuming

$$
\begin{aligned}
& \eta_{i}(t, x)=\left(\sum_{j=1}^{n} q_{i j}(t)\left|x_{j}\right|+q_{i}(t,\|x\|)+1\right)^{-1}\left(f_{i}(t, x)-\sum_{j=1}^{n} p_{0 i j}(t) x_{j}\right), \\
& p_{i j}(t, x)=p_{0 i j}(t)+q_{i j}(t) \eta_{i}(t, x) \operatorname{sgn} x_{j} \quad(i, j=1, \ldots, n)
\end{aligned}
$$

and

$$
\begin{aligned}
& \xi_{l i}(x)=\left(\sum_{j=1}^{n} h_{l i j}(t)\left|x_{j}\right|+h_{l i}(\|x\|)+1\right)^{-1}\left(\iota_{l i}(x)-\sum_{j=1}^{n} \gamma_{0 l i j} x_{j}\right), \\
& \gamma_{l i j}(x)=\gamma_{0 l i j}+h_{l i j} \xi_{l i}(x) \operatorname{sgn} x_{j} \quad\left(i, j=1, \ldots, n ; l=1, \ldots, m_{0}\right),
\end{aligned}
$$

in view of (1.20) and (1.21), respectively, we find

$$
\begin{aligned}
& \left|\eta_{i}(t, x)\right|<1, \quad\left|f_{i}(t, x)-\sum_{j=1}^{n} p_{i j}(t, x) x_{j}\right| \leq q_{i}(t,\|x\|)+1, \\
& p_{0 i j}(t)-q_{i j}(t) \leq p_{i j}(t, x) \leq p_{0 i j}(t)+q_{i j}(t) \quad(i, j=1, \ldots, n)
\end{aligned}
$$

and

$$
\begin{aligned}
& \left|\xi_{l i}(x)\right|<1, \quad\left|\iota_{l i}(x)-\sum_{j=1}^{n} \gamma_{l i j}(x) x_{j}\right| \leq h_{l i}(\|x\|)+1, \\
& \gamma_{0 l i j}-h_{l i j} \leq \gamma_{l i j}(x) \leq \gamma_{0 l i j}+h_{l i j} \quad\left(i, j=1, \ldots, n ; l=1, \ldots, m_{0}\right)
\end{aligned}
$$


where

$$
\begin{aligned}
& P(t, x)=\left(p_{i j}(t, x)\right)_{i, j=1}^{n}, \quad P_{1}(t)=P_{0}(t)-Q(t), \quad P_{1}(t)=P_{0}(t)+Q(t), \\
& J_{l}(x)=\left(\gamma_{l i j}(x)\right)_{i, j=1}^{n}, \quad J_{1 l}=J_{0 l}-H_{l}, \quad J_{2 l}=J_{0 l}+H_{l} \quad\left(l=1, \ldots, m_{0}\right),
\end{aligned}
$$

and

$$
\alpha(t, \rho)=\|q(t, \rho)\|+n \quad \text { and } \quad \beta_{l}(\rho)=\left\|h_{l}(\rho)\right\|+n .
$$

In addition, $P \in \operatorname{Car}^{0}\left([a, b] \times \mathbb{R}^{n}, \mathbb{R}^{n \times n}\right)$. On the other hand, the problem (1.7), (1.8); (1.9) has only the trivial solution for every matrix function $A \in L\left([a, b] ; \mathbb{R}^{n \times n}\right)$ and constant matrices $G_{l} \in \mathbb{R}^{n \times n}\left(l=1, \ldots, m_{0}\right)$, satisfying, respectively, the inequalities (1.18) and (1.19), since the problem (1.24), (1.25); (1.9) has only the trivial solution. Therefore, the theorem follows from Theorem 1.2.

Corollary 1.1 immediately follows from Theorem 1.3 if we assume therein $Q(t) \equiv O_{n \times n}$ and $H_{l}=O_{n \times n}\left(l=1, \ldots, m_{0}\right)$.

To prove Corollaries 1.2-1.5 it is sufficient to show that the problem (1.31), (1.32) has only the trivial solution under the condition $\ell(x)=0$. But this fact is valid, respectively, due to Theorem 3.2, Theorem 3.4, Theorem 3.5, and Corollary 3.2 from [10].

Proof of Theorem 1.4 The solvability of the problem (1.1), (1.2); (1.3) follows from Theorem 1.3, because its conditions are fulfilled for

$$
q(t, \rho) \equiv|f(t, 0)|, \quad h_{l}(\rho) \equiv\left|I_{l}(0)\right| \quad\left(l=1, \ldots, m_{0}\right) \quad \text { and } \quad l_{1}(\rho) \equiv|h(0)| .
$$

Let now $x$ and $y$ be two solutions of the problem (1.1), (1.2); (1.3). Then by (1.36)-(1.38) the vector function $z(t) \equiv x(t)-y(t)$ will be a solution of the problem (1.24), (1.25); (1.9). But this problem has only the trivial solution. Therefore, $x(t) \equiv y(t)$. The theorem is proved.

\section{Competing interests}

The authors declare that they have no competing interests.

\section{Authors' contributions}

The main results have been obtained by MA, and the corollaries have been obtained by GE and NK.

\section{Acknowledgements}

The present paper was supported by the Shota Rustaveli National Science Foundation (Grant \# FR/182/5-101/11).

Received: 29 January 2014 Accepted: 12 June 2014 Published online: 23 September 2014

\section{References}

1. Conti, R: Problèmes linéaires pour les équations différentielles ordinaires. Math. Nachr. 23, 161-178 (1961)

2. Kiguradze, IT: Boundary value problems for systems of ordinary differential equations. In: Current Problems in Mathematics. Newest Results. Itogi Nauki i Tekhniki, vol. 30, pp. 3-103, 204. Akad. Nauk SSSR, Vsesoyuz. Inst. Nauchn. i Tekhn. Inform., Moscow (1987) (in Russian). Translated in J. Sov. Math. 43(2), 2259-2339 (1988)

3. Kiguradze, I, Půža, B: Conti-Opial type existence and uniqueness theorems for nonlinear singular boundary value problems. Funct. Differ. Equ. 9(3-4), 405-422 (2002)

4. Kiguradze, I, Půža, B: On the solvability of nonlinear boundary value problems for functional-differential equations. Georgian Math. J. 5(3), 251-262 (1998)

5. Opial, Z: Linear problems for systems of nonlinear differential equations. J. Differ. Equ. 3, 580-594 (1967) 
6. Akhalaia, S, Ashordia, M, Kekelia, N: On the necessary and sufficient conditions for the stability of linear generalized ordinary differential, linear impulsive and linear difference systems. Georgian Math. J. 16(4), 597-616 (2009)

7. Ashordia, MT: Solvability of quasilinear boundary value problems for systems of generalized ordinary differential equations. Bull. Georgian Acad. Sci. 133(2), 261-264 (1989) (in Russian)

8. Ashordia, MT: Conditions for the existence and uniqueness of solutions of nonlinear boundary value problems for systems of generalized ordinary differential equations. Differ. Uravn. 32(4), 441-449 (1996) (in Russian). Translation in Differ. Equ. 32(4), 442-450 (1996)

9. Ashordia, M: On the correctness of nonlinear boundary value problems for systems of generalized ordinary differential equations. Georgian Math. J. 3(6), 501-524 (1996)

10. Ashordia, M: On the general and multipoint boundary value problems for linear systems of generalized ordinary differential equations, linear impulse and linear difference systems. Mem. Differ. Equ. Math. Phys. 36, 1-80 (2005)

11. Agarwal, RP, O'Regan, D: Multiple nonnegative solutions for second order impulsive differential equations. Appl. Math. Comput. 114(1), 51-59 (2000)

12. Ashordia, M: On the two-point boundary value problems for linear impulsive systems with singularities. Georgian Math. J. 19(1), 19-40 (2012)

13. Ashordia, M, Ekhvaia, G: Criteria of correctness of linear boundary value problems for systems of impulsive equations with finite and fixed points of impulses actions. Mem. Differ. Equ. Math. Phys. 37, 154-157 (2006)

14. Ashordia, M, Ekhvaia, G: On the solvability of a multipoint boundary value problem for systems of nonlinear impulsive equations with finite and fixed points of impulses actions. Mem. Differ. Equ. Math. Phys. 43, 153-158 (2008)

15. Baǐnov, DD, Simeonov, PS: Systems with Impulse Effect. Stability, Theory and Applications. Ellis Horwood Series: Mathematics and Its Applications. Ellis Horwood, Chichester (1989)

16. Benchohra, M, Henderson, J, Ntouyas, S: Impulsive Differential Equations and Inclusions. Contemporary Mathematics and Its Applications, vol. 2. Hindawi Publishing Corporation, New York (2006)

17. Lakshmikantham, V, Bainnov, DD, Simeonov, PS: Theory of Impulsive Differential Equations. Series in Modern Applied Mathematics, vol. 6. World Scientific, Teaneck (1989)

18. Perestyuk, NA, Plotnikov, VA, Samoilenko, AM, Skripnik, NV: Differential Equations with Impulse Effects: Multivalued Right-Hand Sides with Discontinuities. de Gruyter Studies in Mathematics, vol. 40. de Gruyter, Berlin (2011)

19. Samoǐlenko, AM, Perestyuk, NA: Impulsive Differential Equations. World Scientific Series on Nonlinear Science. Series A: Monographs and Treatises, vol. 14. World Scientific, River Edge (1995)

doi:10.1186/s13661-014-0157-8

Cite this article as: Ashordia et al.: On the solvability of general boundary value problems for systems of nonlinear impulsive equations with finite and fixed points of impulse actions. Boundary Value Problems 2014 2014:157.

\section{Submit your manuscript to a SpringerOpen ${ }^{\circ}$ journal and benefit from:}

- Convenient online submission

Rigorous peer review

- Immediate publication on acceptance

- Open access: articles freely available online

- High visibility within the field

- Retaining the copyright to your article 